\title{
On Transitioning From Type-1 to Interval Type-2 Fuzzy Logic Systems
}

\author{
Jabran Hussain Aladi, Student Member, IEEE, Christian Wagner, Senior Member, IEEE, \\ Jonathan M. Garibaldi, Member, IEEE, Amir Pourabdollah, Member, IEEE
}

\begin{abstract}
Capturing the uncertainty arising from system noise has been a core feature of fuzzy logic systems (FLSs) for many years. This paper builds on previous work and explores the methodological transition of type-1 (T1) to interval type-2 fuzzy sets (IT2 FSs) for given "levels" of uncertainty. Specifically, we propose to transition from T1 to IT2 FLSs through varying the size of the Footprint Of Uncertainty (FOU) of their respective FSs while maintaining the original FS shape (e.g., triangular) and keeping the size of the FOU over the FS as constant as possible. The latter is important as it enables the systematic relating of FOU size to levels of uncertainty and vice versa, while the former enables an intuitive comparison between the T1 and T2 FSs. The effectiveness of the proposed method is demonstrated through a series of experiments using the well-known Mackey-Glass (MG) time series prediction problem. The results are compared with the results of the IT2 FS creation method introduced in [1] which follows a similar methodology as the proposed approach but does not maintain the membership function (MF) shape.
\end{abstract}

\section{INTRODUCTION}

The concept of type-2 fuzzy sets (T2 FSs) was introduced by Zadeh in 1975 [2]. Since then, it can be seen that the use of T2 FSs using fuzzy logic system (FLS) to deal with uncertainty appeared in many applications. As more complex models, T2 FSs are considered to be suitable for modelling uncertainty as T2 FSs include Footprint of Uncertainty (FOU) and a third dimension, offering extra degrees of freedom to T2 FSs in comparison to T1 FSs [3], [4].

The computational complexities of using T2 FLSs such as investigated in [5]-[11] have led to the introduction and increased use of the simplified interval type-2 fuzzy logic systems (IT2 FLSs) which today are the most commonly used kind of T2 FLS. IT2 FLSs employ IT2 FSs, which are a special case of a general T2 FSs where all the secondary membership grades are equal to one.

A number of common types of Membership Functions (MFs) for FSs exist in the literature, e.g., triangular, Gaussian, trapezoidal, sigmoidal, pi-shaped, etc. The FOU is a useful concept in IT2 FSs due to the fact that MFs of an IT2 FS are completely described by its FOU [12]. In the literature considering IT2 FLSs, there are many methods used to define an FOU. For example, [13]-[15] apply blurring to the T1 MFs while [16] introduces variations to the T1 MFs to generate IT2 MF. A construction of symmetric or non-symmetric FOUs was proposed in [17]-[19], where each lower membership

The authors are with the Intelligent Modelling and Analysis Group, School of Computer Science, University of Nottingham, Nottingham, UK. (E-mails: itxjha@nottingham.ac.uk, christian.wagner@nottingham.ac.uk, jon.garibaldi@nottingham.ac.uk, amir.pourabdollah@nottingham.ac.uk.)

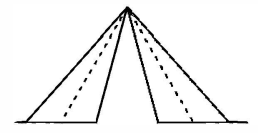

(a)

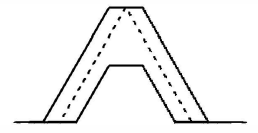

(b)

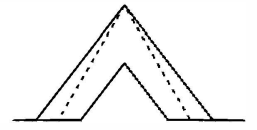

(c)
Fig. 1. Different IT2 FSs around a T1 principal MF (dashed). (a) Conventional IT2 FSs. (b) IT2 FS created using M1. (c) IT2 FS created using M2.

function (LMF) and upper membership function (UMF) used is a standard T1 MF such as triangular, trapezoid, Gaussian. IT2 FSs have also been modelled as geometric objects [20] by utilizing two poly-lines, one used for the UMF of the set and one for the LMF of the set. Ellipsoidal IT2 MFs were created in [21] by introducing an additional parameter into triangular T1 MFs. Diamond shaped MFs [22] were proposed which have certain values at the endpoints while the middle of the MFs captures uncertainty. IT2 FS has also been created in [23], where the left endpoint and the right endpoint of the LMF and UMF are identical to the T1 FS while the FOU is created using $c$ parameter of the triangular IT2 FSs. Finally, IT2 FSs with a uniform FOU over the "core" (support of the LMF) of the fuzzy set, based on a fixed parameter $c \in[0,1]$ which is used to create an FOU of a given size around a (principal) T1 MF was proposed in [1]. To illustrate the context and focus of this paper, we show a number of potential methods for the creation of an IT2 FS by expanding an FOU around a principal T1 MF in Fig. 1. Fig. 1(a) shows a common blurring method which preserves the original $\mathrm{T} 1$ MF, but for which the size of the FOU (i.e. the width of a vertical slice for a given $x$ ) does not maintain constant. This is unintuitive in many applications, in particular when the mound of uncertainty for a given variable (e.g., a sensor) is know in advance - we may not expect that the uncertainty varies in the degree of membership, in particular as strongly as shown in Fig. 1(a). Fig. 1(b) shows an alternative approach presented in [1] which does preserve a uniform/constant level of uncertainty throughout the support of the LMF of the FS, but does not maintain the shape of the original T1 MF. We will refer to this approach as Method 1 (M1) throughout the paper. Finally, in Fig. 1(c), the uncertainty in memberships over the LMF is constant and at the same time, the LMF and UMF keep their original T1 MF shape (dashed line). Thus, the latter approach enables both the adaptation of the IT2 FS for known levels of uncertainty (i.e. by increasing FOU size with increasing uncertainty) and the systematic comparison to the original (in this case triangular) T1 FLS to the resulting new IT2 FLS(s). 
We refer to this last method as Method 2 (M2). The paper focusses on detailing Method 2 and contrasting the resulting IT2 FSs with those resulting when Method 1 is applied in order to highlight potential advantages and disadvantages of both approaches in theoretical work and practical applications.

In summary, in this paper, a new approach M2 is developed to create an FOU of IT2 FS from an original T1 FS by adding a bound value of uncertainty to the original T1 MFs parameters and scaling the LMF by a fixed parameter to ensure that the resulting FOU is uniform within the core (i.e. the support of the lower MF) of the IT2 FSs. This IT2 FS creation method is proposed to maintain the level of uncertainty captured in the primary memberships of the FS constant, while also keeping the IT2 FS shape (LMF and UMF) the same as the original T1 MF. The latter differentiates the proposed method from M1 presented in [1], which starts for example with a triangular shape in T1 principal MFs and as we increase the FOU size, the shape of IT2 FS changes to different shape (i.e., trapezoidal) (see Fig. 1(b)).

The rest of the paper will cover the following. In Section II, a review of T2 FSs, singleton fuzzy logic system (SFLSs) and non-singleton fuzzy logic system (NSFLS), IT2 FS generation techniques and additive noise are provided. The developed method is explained further in Section III. Both methods (M1 and M2) are applied to MG time series and presented in Section IV. The results and discussions of the study are presented in Section V. The conclusion and future work appear in Section VI.

\section{BACKGROUND}

This section provides a brief overview of the concepts used later in the paper. These concepts include T2 FSs, SFLS and NSFLSs, IT2 FS generation techniques and noise levels or more specifically, the Signal-to-Noise Ratio (SNR) which is employed throughout the paper as a measure of uncertainty.

\section{A. Type-2 fuzzy sets}

T2 FSs were originally proposed by Zadeh [2] and are an extension of T1 FSs. A T2 Fs, $\tilde{A}$, may be represented as [6]:

$$
\tilde{A}=\left\{\left((x, u), \mu_{\tilde{A}}(x, u)\right) \mid \forall x \in X, \forall u \in J_{x} \subseteq[0,1]\right\},
$$

where $J_{x}$ is the primary membership of $x$ and $\mu_{\tilde{A}}(x, u) \in$ $[0,1]$ is the secondary MF. $\tilde{A}$ in (1) can also be defined as:

$$
\tilde{A}=\int_{x \in X} \int_{u \in J_{x}} \mu_{\tilde{A}}(x, u) /(x, u) J_{x} \subseteq[0,1],
$$

where $\int$ denotes union and is replaced by $\sum$ if the universe of discourse is discrete.

When all the secondary grades $\mu_{\tilde{A}}(x, u)$ are equal to one, then, $\tilde{A}$ is an IT2 FS. Following (2), an IT2 FS $\tilde{A}$ can be expressed as [3]:

$$
\tilde{A}=\int_{x \in X} \int_{u \in J_{x}} 1 /(x, u) J_{x} \subseteq[0,1]
$$

The domain of the primary membership $J_{x}$ defines the FOU of FS $\tilde{A}$ [3]:

$$
\operatorname{FOU}(\tilde{A})=\bigcup_{\forall x \in X} J_{x}
$$

Alternatively, the FOU of an IT2 FS $\tilde{A}$ can be described by its LMFs and UMFs [4]:

$$
\operatorname{FOU}(\tilde{A})=\bigcup_{\forall x \in X}\left[\underline{\mu}_{\tilde{A}}(x), \bar{\mu}_{\tilde{A}}(x)\right]
$$

\section{B. Singleton and non-singleton type-2 fuzzy logic system}

According to the type of fuzzification [3], IT2 FLSs can be divided into SFLS and NSFLS, which are presented below. T2 FLSs are the extension of the T1 FLSs. The MFs of T2 FLSs in the antecedents and/or the consequents are T2 FSs [24].

An IT2 FLS consists of five components, which are fuzzifier, rule base, inference engine, type reducer, and defuzzifier. In singleton IT2 FLS, crisp inputs are first fuzzified, usually into input IT2 FSs (in singleton fuzzification). These activate the inference engine and the rule base to produce output IT2 FSs which are then combined to produce an aggregated IT2 output FSs. They are then processed by a type-reducer such as the centroid type-reducer, which performs a centroid calculation, leading to T1 FSs known as type-reduced set(s) [3]. The defuzzifier finally defuzzifies the type-reduced type1 fuzzy outputs to produce crisp outputs. Further detail on SFLSs can be found for example in [3], [24].

A non-singleton IT2 FLS has the same structure as a singleton IT2 FLS, and they share the same type of rules; the major difference is the type of fuzzification. The majority of FLSs are using SFLS because the singleton fuzzification is simpler and faster to compute. In singleton fuzzification, inputs are considered to be singleton FSs, while the non-singleton fuzzification models the FLS inputs as FSs. For more details on NSFLSs can be found for example in [3], [25], [26].

\section{Interval type-2 fuzzy set generation techniques}

IT2 FSs can have different shapes such as triangular or trapezoidal shapes. To make a general case for FOU creation techniques, we will use a common method such as Gaussian IT2 FS with uncertain parameters for transition $(t)$, scaling $(s)$, mean $(m)$ and standard deviation $(\sigma)$ introduced in [27] as:

$$
\begin{aligned}
& \mu(x)=s * e^{-\frac{1}{2}\left(\frac{x-m}{\sigma}\right)^{2}}+t, \\
& \quad s \in\left[s_{1}, s_{2}\right], m \in\left[m_{1}, m_{2}\right], \sigma \in\left[\sigma_{1}, \sigma_{2}\right], t \in\left[t_{1}, t_{2}\right]
\end{aligned}
$$

Here, a similar methodology applied for triangular and trapezoidal IT2 FSs. In case of a triangular FS with uncertain parameters for transition $(t)$, scaling $(s)$, left end point $(a)$, centre $(m)$ and right end point $(b)$ can be defined as:

$$
\begin{array}{r}
\mu(x)=s *\left\{\begin{array}{cc}
0 & b \leq x \leq a \\
\frac{x-a}{m-a} & a \leq x \leq m \\
\frac{b-x}{b-m} & m \leq x \leq b
\end{array} \quad+t,\right. \\
s \in\left[s_{1}, s_{2}\right], a \in\left[a_{1}, a_{2}\right], b \in\left[b_{1}, b_{2}\right], m \in\left[m_{1}, m_{2}\right], \\
t \in\left[t_{1}, t_{2}\right]
\end{array}
$$

In case of a trapezoidal FS with uncertain parameters for transition $(t)$, scaling $(s)$, left end point $(a)$, left centre $\left(m_{1}\right)$, 
right centre $\left(m_{2}\right)$ and right end point $(b)$ can be defined as:

$$
\begin{aligned}
& \mu(x)=s *\left\{\begin{array}{cc}
0 & b \leq x \leq a \\
\frac{x-a}{m_{1}-a} & a \leq x \leq m_{1} \\
1 & m_{1} \leq x \leq m_{2} \\
\frac{b-x}{b-m_{2}} & m_{2} \leq x \leq b
\end{array}+t\right. \\
& s \in\left[s_{1}, s_{2}\right], a \in\left[a_{1}, a_{2}\right], b \in\left[b_{1}, b_{2}\right], m_{1} \in\left[m_{1_{1}}, m_{1_{2}}\right] \text {, } \\
& m_{2} \in\left[m_{2_{1}}, m_{2_{2}}\right], t \in\left[t_{1}, t_{2}\right]
\end{aligned}
$$

In (6-8), LMF and UMF $\underline{\mu}(x)$ and $\bar{\mu}(x)$ can be obtained by varying one or more parameters and fixing the other parameters. Also, note if the transition parameter $(t)$ is set to 0 and the scaling parameter $(s)$ is set to 1 and the other parameters are made fixed then the MFs become T1 MFs. In the following subsection, we will briefly review the method M1 initially presented in [1].

1) FOU creation method (M1): As previously introduced in [1], this method is used to obtain an IT2 FSs with a uniform FOU over the core of the FS, where an FOU construction method based on a fixed parameter $c$ is used to create an FOU of a given size around a principal (T1) MF. In order to create the IT2 FSs based on the uncertainty parameter $c$ and the T1 MF, we employ (9) and (10) shown below to create the resulting UMF and LMF respectively.

$$
\begin{gathered}
\bar{\mu}(x)=\min \left(\mu(x)+\frac{c}{2}, 1.0\right) \\
\underline{\mu}(x)=\min \left(\max \left(\mu(x)-\frac{c}{2}, 0\right), 1.0-c\right),
\end{gathered}
$$

where $\mu(x)$ relates to the T1 MFs in (7) for triangular FS and (8) for trapezoidal FS. Note the parameter $t$ in both (7) and (8) is set to $\frac{c}{2}$ for $\bar{\mu}(x)$ in (9) and $-\frac{c}{2}$ for $\underline{\mu}(x)$ in (10). The parameter $s$ is set to 1 , whereas no changes to the other parameters are applied. For more details on this method we refer the reader to [1]. A more detailed illustration of the M1 design of the IT2 FS is depicted in Fig. 2(a) and 2(b) - for triangular MFs and Fig. 3(a) and 3(b) - for trapezoidal MFs.

\section{Additive noise}

In engineering applications, the level of noise is commonly measured by the SNR (see (11)) where a high SNR refers to a clear signal (low noise) and a low SNR refers to a noisy signal (high amounts of noise). As a common and welldefined measure of (one type of) uncertainty, we will use SNR throughout in this paper. In our experiments, $h$ different levels of a given SNR and noise which has a uniform distribution with zero-mean and $\sigma_{\text {noise }}$ is injected into the training and the testing sets of existing samples of the MG time series. The formula for SNR (in dBs) [3]:

$$
S N R=10 * \log _{10}\left(\frac{\sigma^{2} \text { signal }}{\sigma^{2} \text { noise }}\right)
$$

where $\sigma^{2}$ signal is the variance of the signal and $\sigma^{2}$ noise is the variance of the noise. To find $\sigma_{\text {noise }}$ we solve (11) for $\sigma_{\text {noise }}$ as

$$
\sigma_{\text {noise }}=\frac{\sigma_{\text {signal }}}{10^{\left(\frac{\text { SNR }}{20}\right)}}
$$

Then, the noise value $n(t)$ can be generated using a uniform random variable with zero-mean and $\sigma_{\text {noise }}$ at different SNR values and then added to the time series $x(t)$.

\section{A NOVEL FOU CREATION METHOD (M2)}

As discussed in Section I, in an effort to find an alternative FOU creation method, addressing the shortcoming of M1 which starts for example with a triangular shape in T1 principal MFs and as we increase the FOU size, the shape of IT2 FS changes to different shape (i.e., trapezoidal), a new method is proposed. This novel FOU creation method (M2) is used to obtain an IT2 FSs with a uniform FOU that captures a constant level of uncertainty over the core of the fuzzy set based on a bounded values of uncertainty $\delta$ and a fixed parameter $c \in[0,1]$. The parameters are used to create an FOU of a given size around a (principal) T1 MF and maintaining the same shape of $\mathrm{T} 1 \mathrm{MF}$ for both (UMF and LMF) as the FOU increases.

We start the design of the IT2 FSs by including the FOU size parameter $c$ and the bounded values of uncertainty $\delta$ to form the IT2 FSs of the system. Note that when both $c$ and $\delta$ are equal to zero, then the result is the original T1 FS MF, while $c=1$ results in an IT2 set with a very wide FOU (as detailed further below).

In order to preserve the original shape of the membership function (T1 MF) as we add a certain value of the FOU size parameter $c$ to it to create an IT2 FSs, we construct the UMF, $\bar{\mu}(x)$ and the LMF, $\mu(x)$ of the IT2 FSs by adding/subtracting a specific value of $(\bar{\delta})$ from the T1 MF parameters as follows:

$$
\begin{aligned}
\underline{a} & =a+\delta \\
\bar{a} & =a-\delta \\
\underline{b} & =b-\delta \\
\bar{b} & =b+\delta
\end{aligned}
$$

where $\underline{a}$ and $\underline{b}$ are the left and the right points of the LMFs, $\bar{a}$ and $\bar{b}$ are the left and the right points of the UMFs. The UMF, $\bar{\mu}(x)$ and the LMF, $\mu(x)$ of IT2 FS for triangular case can then be obtained as follows:

$$
\begin{aligned}
& \bar{\mu}(x)=\left\{\begin{array}{cc}
0 & \bar{b} \leq x \leq \bar{a} \\
\frac{x-\bar{a}}{m-\bar{a}} & \bar{a} \leq x \leq m \\
\bar{b}-x & m \leq x \leq \bar{b}
\end{array}\right. \\
& \underline{\mu}(x)=(1-c) * \begin{cases}0 & \underline{b} \leq x \leq \underline{a} \\
\frac{x-\underline{a}}{m-\underline{a}} & \underline{a} \leq x \leq m \\
\underline{\underline{b}-x} & m \leq x \leq \underline{b}\end{cases}
\end{aligned}
$$

Whereas, for trapezoidal case, The UMF, $\bar{\mu}(x)$ and the LMF, $\underline{\mu}(x)$ of IT2 FS can then be obtained as follows:

$$
\bar{\mu}(x)=\left\{\begin{array}{cc}
0 & \bar{b} \leq x \leq \bar{a} \\
\frac{x-\bar{a}}{m_{1}-\bar{a}} & \bar{a} \leq x \leq m_{1} \\
1 & m_{1} \leq x \leq m_{2} \\
\frac{\bar{b}-x}{\bar{b}-m_{2}} & m_{2} \leq x \leq \bar{b}
\end{array}\right.
$$




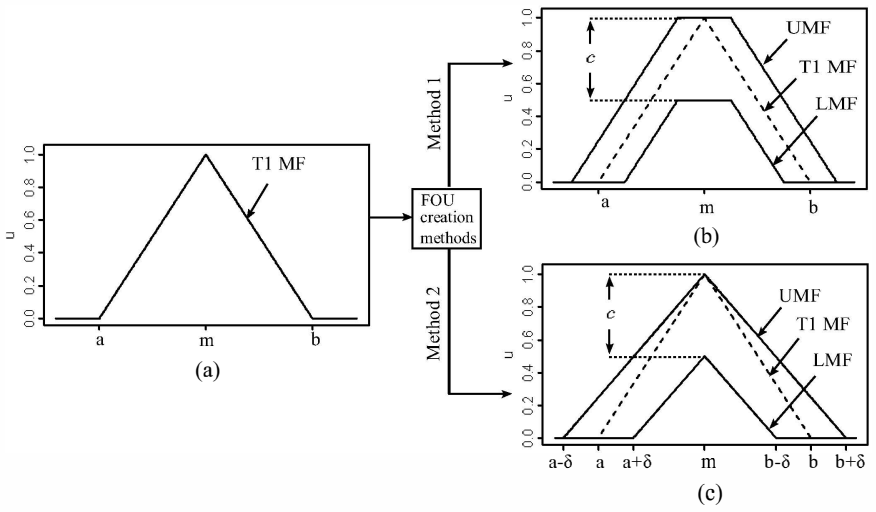

Fig. 2. An illustration of design methods of triangular IT2 MFs with FOU size parameter $c=0.5$. (a) Initial T1 FS, (b) IT2 using M1 and (c) IT2 FS using M2.

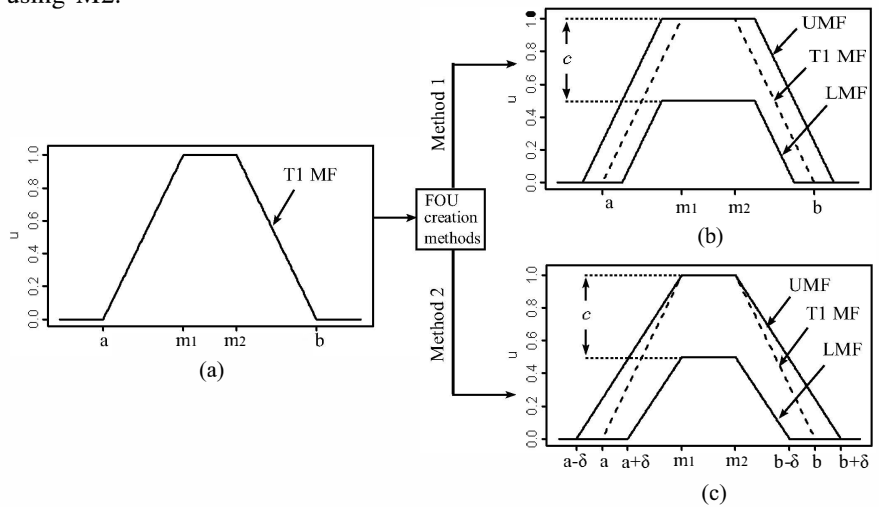

Fig. 3. An illustration of design methods of trapezoidal IT2 MFs with FOU size parameter $c=0.5$. (a) Initial T1 FS, (b) IT2 using M1 and (c) IT2 FS using M2.

$$
\underline{\mu}(x)=(1-c) *\left\{\begin{array}{cc}
0 & \underline{b} \leq x \leq \underline{a} \\
\frac{x-\underline{a}}{m_{1}-\underline{a}} & \underline{a} \leq x \leq m_{1} \\
1 & m_{1} \leq x \leq m_{2} \\
\underline{b-x} & m_{2} \leq x \leq \underline{b}
\end{array}\right.
$$

The parameter $(t)$ in both (7) and (8) has become 0 in (16) and (17). The parameter $s$ has become 1 for $\bar{\mu}(x)$ in (16) and $s=(1-c)$ for $\underline{\mu}(x)$ in (16) and no changes were made to the other parameters for both cases. In (15) and (17) we scaled the lower membership function by $(1-c)$, to create a uniform FOU all over the IT2 FS. Parameter $c \in[0,1]$ is the FOU size parameter. The $\delta$ parameter is used to define the endpoints in the fuzzy MFs (see Fig. 2 and Fig. 3). Its value can be obtained experimentally, so that, for a given value of $c$, the uncertainty indicator, $U(x)$ in (18) below, is constant (equal to $c$ ) over the support of the LMF $(\mu(x)>0)$ ). A more detailed illustrations of design of the IT2 FS is depicted in Fig. 2(a) and 2(c) for triangular MFs and Fig. 3(a) and 3(c) for trapezoidal MFs using M2.

$$
U(x)=\bar{\mu}(x)-\underline{\mu}(x)
$$

After considering the proposed FOU creation techniques, we proceed to an experimental exploration of the different behaviour of IT2 FSs created using either M1 or M2 when transitioning from a T1 to an IT2 FLS.

\section{TRANSITIONING FROM T1 TO ITS FLSS}

This section describes the design of the T1 FLS for a given application and its subsequent transformation to one or more IT2 FLSs. In order to study the behaviour of the proposed FOU creation technique presented in this paper, a systematic methodology for the transitioning from T1 to IT2 FLSs is presented. The designs of T1/IT2 FLSs will be considered with respect to different noise levels and thus different desired FOU sizes. The complete process can be summarised in four steps as follows:

\section{A. Data Generation}

First, training and testing data from the system under study (e.g., a time series) is generated. The training and testing data is injected with different noise levels. The training data is used to train the system (generating rules) under two conditions: noise free data (NFtrain) and noisy data (Ntrain) at different noise levels. The testing data sets (noise free (NFtest and corrupted by noise) are used to test the performance of a designed system in the face of a given level of noise.

\section{B. T1 FSs design and rule-base creation}

Next, T1 FSs are created, either by an expert (as further detailed in Section V) or through an automatic method (e.g., a genetic algorithm). Second, the training data is used to generate the rules using for example the WM-method [28].

\section{IT2 FSs Design (FOU Construction)}

The T1 FSs are extended to IT2 FSs using the existing T1 MFs as a basis. For the FOU size parameters $c \in[0,1]$, $l$ values are chosen. Also, a number $h$ of noise/uncertainty levels are chosen for investigation. For M1, the UMFs and LMFs will be constructed using (9) and (10) respectively. For M2, upper and lower triangular MFs will be constructed using (14) and (15) and upper and lower trapezoidal MFs will be constructed using (16) and (17) respectively. The $\delta$ parameter is used to define the bounded values of uncertainty in the fuzzy MFs and its values are obtained experimentally, as discussed in Section III. This enables the evaluation of each one of the FLSs for $h$ different noise levels (and FOU sizes) for both methods. For all FLSs, both SFLS and NSFLS versions are evaluated.

\section{FLS Performance Testing and Evaluation}

After finishing the design of each of the IT2 FLS with the chosen FOU size parameter, we test its performance using the pre-generated testing data at each of the $h$ noise/uncertainty levels. At each noise level, the performance testing is repeated a number of times (in our case: 30 times) to account for the random character of the noise injection. The performance of the design(s) is evaluated, for example using the Root Mean-Squared Error (RMSE). The average of the RMSEs is then calculated over all iterations for each FLS at each noise/uncertainty level in order to enable the best mapping between the FLSs (with different FOU sizes) and the noise 
levels. This is valuable in order to determine which FOU size of which design method (M1 or M2) provides the best results in the face of different levels of uncertainty (in our case: SNR).

In the following section we conduct a set of experiments using the FLS design described to compare and contrast M1 and M2 in the context of well-known MG time series.

\section{EXPERIMENTS}

FLSs have been successfully used in forecasting of time series [3], [28]-[31]. As the level of noise/uncertainty is easily controllable, we use time-series prediction here as a test bed to explore the different approaches to IT2 FLS generation. We use the Mackey-Glass time series which is a chaotic time series proposed in [32]. It is a first-order differential-delay equation used to model physiological systems. It is generated from the following non-linear differential equation:

$$
\frac{d x(t)}{d t}=\frac{a * x(t-\tau)}{1+x^{n}(t-\tau)}-b * x(t)
$$

where $a, b$ and $n$ are constant real numbers, $t$ is the current time and $\tau$ is the delay time. For $\tau \leq 17$, the system is known to exhibit a deterministic/periodic behaviour which turns chaotic with $\tau>17$. To obtain simulation data, (19) is used in this paper with the following parameters: $a=0.2$, $b=0.1, \tau=30$ and $n=10$ and solved using Euler's method [33] with a step size equal to 1.0 and the initial values of $x(t)$ for all values of $t \leq \tau$ are set to 0.9 . The detail of the steps is given below.

\section{A. Data Generation}

Noise-free data are generated using (19) for the MG timeseries. To obtain training and testing input-output data pairs, we extract 700 input-output data pairs. In our case, four-input, one-output FLSs (single stage prediction) are considered. The training dataset is the first $\mathbf{5 0 0}$ data points used for raining the FLSs (generating the rules). The following 200 points are used for testing the FLSs. In this paper, we consider different versions of training and testing data which are corrupted with zero-mean uniform noise for different SNRs. In this work we used 5 noise levels in training and the same number in testing (i.e. $h=5$ ). The chosen noise levels are (from lower to higher level): 20,16,10,4 and $0 \mathrm{dBs}$. The original noise free (NF) data also used for training and testing the FLSs.

\section{B. T1 FSs design and rule-base creation}

In this paper, we choose the number of membership function (both triangular and trapezoidal) for each input and output of the FLS to be 7 MFs which are defined evenly to cover the input and output spaces. Then, we apply the well-known WM method [28] to create the rules for the given training inputoutput dataset. The result is 12 rule bases for the different combinations as shown in Table I. The actual number of FSs and the rules are maintained from the T1 FLSs and used for all resulting IT2 FLSs. From Table I, we can see that both triangular and trapezoidal MFs used in T1 FSs generate the same number of rules at each noise level.
TABLE I

NUMBER OF RULES GENERATED AT EACH NOISE LEVEL USING THE WM-METHOD APPLIED TO T1 TRIANGULAR AND TRAPEZOIDAL FLSS

\begin{tabular}{|c|c|c|}
\hline Noise Level & triangular MF & trapezoidal MF \\
\hline NF & 47 & 47 \\
\hline 20 & 80 & 80 \\
\hline 16 & 91 & 91 \\
\hline 10 & 125 & 125 \\
\hline 4 & 283 & 283 \\
\hline 0 & 330 & 330 \\
\hline
\end{tabular}

TABLE II

$\delta$ VALUES FOR BOTH TRIANGULAR AND TRAPEZOIDAL FS $S$

\begin{tabular}{|c|c|c|c|c|c|}
\hline$c$ & 0 & 0.2 & 0.4 & 0.8 & 1 \\
\hline$\delta$ triangular MF & 0 & 0.02 & 0.045 & 0.12 & 0.182 \\
\hline$\delta$ trapezoidal MF & 0 & 0.015 & 0.033 & 0.088 & 0.127 \\
\hline
\end{tabular}

\section{IT2 FSs Design (FOU Construction)}

Following Section IV.C, we extend the T1 FLSs into a series of IT2 FLSs. First, we design the T1 FSs for both triangular and trapezoidal and create the rule bases using the WM approach using the training data set. Then, we start the design of a series of IT2 FLSs by generating IT2 FSs using the FOU creation methods (M1 and M2) described in Sections II and III to form the IT2 MFs of the systems. The actual number of FSs and the rules are maintained from the T1 system. All the common parameters between the SFLSs and NSFLSs are chosen to be the same. For all experiments, $\sigma_{x}$ in the NSFLS case is set equal to the standard deviation of the additive noise. In a noise free situation for which $\sigma_{x}=0$, the performance of the NSFLS is identical to that of the SFLS.

To construct the upper and lower membership functions of the IT2 FSs, we use the following:

- The chosen FOU parameter $c$ values used for both methods (M1 and M2) are 0,2,4,8,and 1 (i.e. $l=5$ ). At $c=0$ the IT2 FSs reduce to the original T1 FSs, whereas in case of using $c=1$, the IT2 FSs reach the maximum amount of their width.

- For M1, we design the IT2 FSs using (9) and (10) for both triangular and trapezoidal cases.

- For M2, we design the IT2 FSs using (14) and (15) for triangular case and (16) and (17) for trapezoidal cases.

- For M2, the $\delta$ parameter is used to define the bounded values of MFs and its values can be obtained experimentally as shown in Section III. $\delta$ values for both triangular and trapezoidal FSs are shown in Table II.

Table III shows the triangular and trapezoidal FSs at different $c$ values for M1 and M2. Note how both methods maintain a constant level of uncertainty (FOU size) over the support of the LMFs, while M2 also preserves the shape of the T1 MFs. After creating the IT2 FSs (triangular and trapezoidal) for both M1 and M2, we start to design SFLS and NSFLSs using the rule bases generated previously in step B. Each FLS uses product t-norm, product inference, centroid type-reduction and centroid defuzzification.

After finishing the design of the IT2 FLSs with the chosen FOU sizes for both methods at different configurations as mentioned above, the testing data sets are used to test the 
TABLE III

TRIANGULAR AND TRAP. FSS AT DIFFERENT $c$ VALUES FOR M1 AND M2

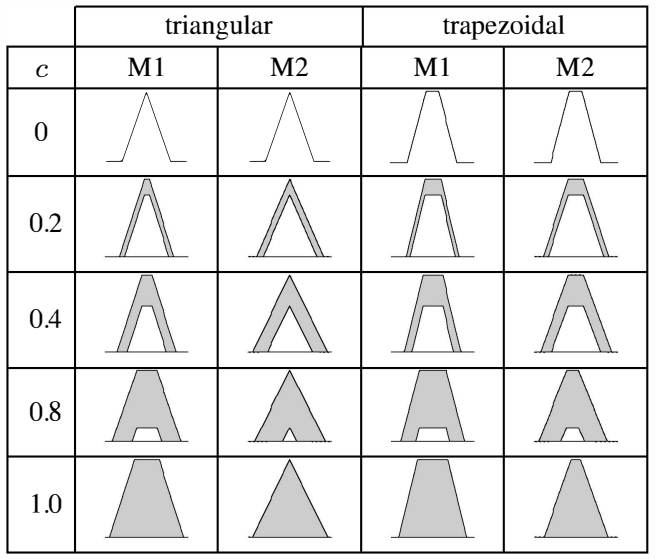

performance of the individual IT2 FLSs when faced with the different uncertainty/noise levels. Now that we have a total of 20 FLSs for each design method with each case of MF case (triangular and trapezoidal) (i.e. 5 levels of $c$ $* 2$ training conditions (NFtrain and Ntrain) * 2 FLS types (NSFLS, SFLS)). The total number of constructed FLSs are thus 80 FLSs (20 with triangular (M1), 20 with triangular (M2) 20 for trapezoidal (M1) and 20 for trapezoidal (M2)) and each system will be tested over 5 different noise levels and the NFtest case. The testing and evaluation of these systems will be presented next.

\section{FLSs Performance Testing and Evaluation}

We test each of the IT2 FLSs described above against 6 levels of noise in order to determine the best performance for each given noise level. Each test is repeated 30 times to account for the random generation of the uniform noise. The performances of all the designs were evaluated using their Root Mean Squared Error (RMSE) over the testing data set:

$$
R M S E=\sqrt{\frac{1}{200} \sum_{t=1508}^{1707}\left[s(t+1)-f\left(\mathbf{s}^{(t)}\right)\right]^{2}}
$$

where, $s(t+1)$ is the output of the noisy testing data and $f\left(s^{(t)}\right)$ is the crisp output of the FLS, and, $\mathbf{s}^{(t)}=$ $[s(t-3), s(t-2), s(t-1), s(t)]^{T}$. The RMSE results are averaged over 30 runs and are depicted in Tables IV and $\mathrm{V}$ showing the results of the average RMSE of the MG time series using the two different MF types (triangular and trapezoidal) with the two different design methods (M1 and M2). Each column represents an IT2 FLS design with a given FOU size parameter $c$ and the rows show the average RMSE value at the different SNR values for all FOU sizes/FLSs. The shaded values are the result of M2.

\section{RESULTS AND DisCUSSION}

In order to explore the viability and effect of the novel method M2, in particular in relation to M1, we briefly discuss the results of the experiments conducted in the previous sections. After analysis of the RMSE results of both methods, we can divide the analysis into three areas. The first analysis covers a comparison of the FLSs based on M1 and M2 in terms of their performance in general. The second analysis will be devoted to the performance of M1 and M2 in different settings (i.e., training on NFtrain and Ntrain data, SFLSs and NSFLSs. The third analysis will include the performance analysis of FLSs with the two cases (triangular and trapezoidal MFs) on different settings. Finally, common observations will be presented.

The average RMSE values for M1 and M2 are depicted in Table IV and V. The results are visualised in Fig. 4. Generally, there are no significant differences between them in the sense that NSFLSs provide superior outputs to SFLSs. Also, training the systems on noisy data produces better performance especially with the SFLSs cases. As we increase the FOU size parameter $c$, the performance gets better. At $c=0$ both methods have the same result as the systems reduce to the original T1 FLS. By calculating the relative percentage between M1 and M2 in the triangular MF case, we have found that M1 achieved better performance reaching around $34 \%$ at $c=0.8$ and $20 \%$ at $c=1.0$. However, the relative percentage between M1 and M2 in case of trapezoidal MF is smaller reaching around $22 \%$ at $c=0.8$ and significantly less for the other $c$ values.

A comparison of $\mathrm{M} 1$ and M2 performance in case of triangular MF is shown in Fig. 5. By comparing the results of both methods we have found that the FLSs trained with noisy data performed better than those trained with NF as can be expected. In case of SFLSs and at low SNR (higher noise levels), FLSs trained with noisy data (Ntrain) are performing better with around $41 \%$ than FLSs trained with NF data (NFtrain). From Fig. 5, it is clear that M1 is performing better than M2 in most of the cases. Overall, as expected, NSFLSs perform much better than SFLSs especially at lower SNR values (higher noise levels). Finally, from the results, the following are observed: 1) All FLSs provide better performance when SNR increases. 2) A direct relationship between the FOU size of the FSs and the noise level has been observed showing that as the noise level increases, the FOU that gives the minimum RMSE value increases as well with better performance. This result is aligned with [1]. 3) Training the systems with noisy data improves the performance of FLSs. 4) NSFLSs outperform their counterparts SFLSs. 5) Triangular and trapezoidal MFs produce the same number of rules at each noise levels. 6) M1 show better performance than M2 especially with triangular MFs.

\section{CONCLUSION}

As part of this paper, we propose a methodological approach to transitioning from T1 to IT2 FSs for different levels of uncertainty (noise), while preserving the original T1 MF shape in the IT2 MFs. Thus, the objective of this work is not to achieve optimal performance in applications such as in time series prediction, but to study and present an IT2 FS creation method that systematically captures a specified amount of uncertainty (i.e., the uncertainty in memberships over the 
TABLE IV

THE AVERAGE RMSE VALUES FOR MG TIME SERIES PREDICTION FOR TRIANGULAR MFS (SHADED VALUES ARE THE RESULTS OF M2)

\begin{tabular}{|c|c|c|c|c|c|c|c|c|c|c|c|c|c|c|c|c|c|c|c|c|}
\hline & \multicolumn{4}{|c|}{$\mathrm{c}=0$} & \multicolumn{4}{|c|}{$\mathrm{c}=0.2$} & \multicolumn{4}{|c|}{$\mathrm{c}=0.4$} & \multicolumn{4}{|c|}{$\mathrm{c}=0.8$} & \multicolumn{4}{|c|}{$\mathrm{c}=1.0$} \\
\hline & \multicolumn{2}{|c|}{ NFtrain } & \multicolumn{2}{|c|}{\begin{tabular}{|l} 
Ntrain \\
\end{tabular}} & \multicolumn{2}{|c|}{$\begin{array}{l}\text { NFtrain } \\
\end{array}$} & \multicolumn{2}{|c|}{$\begin{array}{l}\text { Ntrain } \\
\end{array}$} & \multicolumn{2}{|c|}{$\begin{array}{l}\text { NFtrain } \\
\end{array}$} & \multicolumn{2}{|c|}{ Ntrain } & \multicolumn{2}{|c|}{ NFtrain } & \multicolumn{2}{|c|}{\begin{tabular}{|l} 
Ntrain \\
\end{tabular}} & \multicolumn{2}{|c|}{ NFtrain } & \multicolumn{2}{|c|}{\begin{tabular}{|l} 
Ntrain \\
\end{tabular}} \\
\hline & SFLS & NSFLS & SFLS & NSFLS & SFLS & NSFLS & SFLS & NSFLS & SFLS & NSFLS & SFLS & NSFLS & SFLS & NSFLS & SFLS & NSFLS & SFLS & NSFLS & SFLS & NSFLS \\
\hline \multirow[t]{2}{*}{ NFtest } & 0.0313 & 0.0313 & 0.0313 & 0.0313 & 0.0318 & 0.0318 & 0.0318 & 0.0318 & 0.0369 & 0.0369 & 0.0369 & 0.0369 & 0.0593 & 0.0593 & 0.0593 & 0.0593 & 0.0784 & 0.0784 & 0.0784 & 0.0784 \\
\hline & 0.0313 & 0.0313 & 0.0313 & 0.0313 & 0.0321 & 0.0321 & 0.0321 & 0.0321 & 0.0400 & 0.0400 & 0.0400 & 0.0400 & 0.0679 & 0.0679 & 0.0679 & 0.0679 & 0.0914 & 0.0914 & 0.0914 & 0.0914 \\
\hline \multirow[t]{2}{*}{20} & 0.0426 & 0.0425 & 0.0540 & 0.0539 & 0.0423 & 0.0424 & 0.0538 & 0.0538 & 0.0451 & 0.0451 & 0.0553 & 0.0553 & 0.0610 & 0.0621 & 0.0636 & 0.0642 & 0.0765 & 0.0752 & 0.0771 & 0.0775 \\
\hline & 0.0426 & 0.0425 & 0.0540 & 0.0539 & 0.0424 & 0.0425 & 0.0539 & 0.0539 & 0.0468 & 0.0466 & 0.0562 & 0.0561 & 0.0741 & 0.0735 & 0.0766 & 0.0759 & 0.0897 & 0.0903 & 0.0903 & 0.0921 \\
\hline \multirow[t]{2}{*}{16} & 0.0554 & 0.0551 & 0.0697 & 0.0690 & 0.0549 & 0.0548 & 0.0686 & 0.0680 & 0.0563 & 0.0558 & 0.0683 & 0.0680 & 0.0671 & 0.0669 & 0.0802 & 0.0797 & 0.0800 & 0.0783 & 0.0977 & 0.0960 \\
\hline & 0.0554 & 0.0551 & 0.0697 & 0.0690 & 0.0550 & 0.0548 & 0.0685 & 0.0680 & 0.0570 & 0.0562 & 0.0690 & 0.0686 & 0.0773 & 0.0755 & 0.0955 & 0.0903 & 0.0877 & 0.0901 & 0.0958 & 0.1018 \\
\hline \multirow[t]{2}{*}{10} & 0.0997 & 0.0918 & 0.1039 & 0.1006 & 0.0927 & 0.0904 & 0.0996 & 0.0967 & 0.0917 & 0.0875 & 0.0914 & 0.0898 & 0.0923 & 0.0831 & 0.0840 & 0.0923 & 0.1024 & 0.1004 & 0.1087 & 0.112 \\
\hline & 0.0997 & 0.0918 & 0.1039 & 0.1006 & 0.0929 & 0.0904 & 0.0995 & 0.0967 & 0.0919 & 0.0870 & 0.0908 & 0.0894 & 0.0985 & 0.0867 & 0.1055 & 0.0988 & 0.1000 & 0.1026 & 0.1112 & 0.1175 \\
\hline \multirow[t]{2}{*}{4} & 0.3355 & 0.1526 & 0.1971 & 0.1747 & 0.2639 & 0.1488 & 0.1832 & 0.1664 & 0.1934 & 0.1401 & 0.1603 & 0.1511 & 0.1430 & 0.1239 & 0.1454 & 0.1038 & 0.1487 & 0.1399 & 0.1498 & 0.1611 \\
\hline & 0.3355 & 0.1526 & 0.1971 & 0.1747 & 0.2816 & 0.1494 & 0.1851 & 0.1674 & 0.2135 & 0.1405 & 0.1613 & 0.1512 & 0.1429 & 0.1234 & 0.1467 & 0.1393 & 0.1444 & 0.1359 & 0.1498 & 0.1655 \\
\hline \multirow[t]{2}{*}{0} & 0.5150 & 0.2088 & 0.3023 & 0.2519 & 0.4418 & 0.2039 & 0.2797 & 0.2410 & 0.3714 & 0.1928 & 0.2423 & 0.2191 & 0.2632 & 0.1692 & 0.2024 & 0.1839 & 0.2275 & 0.1819 & 0.2010 & 0.1947 \\
\hline & 0.5150 & 0.2088 & 0.3023 & 0.2519 & 0.4691 & 0.2052 & 0.2854 & 0.2429 & 0.4127 & 0.1948 & 0.2506 & 0.2212 & 0.2789 & 0.1697 & 0.2038 & 0.1835 & 0.2129 & 0.1817 & 0.1991 & 0.1968 \\
\hline
\end{tabular}

TABLE V

THE AVERAGE RMSE VALUES FOR MG TIME SERIES PREDICTION FOR TRAPEZOIDAL MFS (SHADED VALUES ARE THE RESULTS OF M2)

\begin{tabular}{|c|c|c|c|c|c|c|c|c|c|c|c|c|c|c|c|c|c|c|c|c|}
\hline & \multicolumn{4}{|c|}{$c=0$} & \multicolumn{4}{|c|}{$\mathrm{c}=0.2$} & \multicolumn{4}{|c|}{$\mathrm{c}=0.4$} & \multicolumn{4}{|c|}{$\mathrm{c}=0.8$} & \multicolumn{4}{|c|}{$c=1.0$} \\
\hline & \multicolumn{2}{|c|}{ NFtrain } & \multicolumn{2}{|c|}{ Ntrain } & \multicolumn{2}{|c|}{ NFtrain } & \multicolumn{2}{|c|}{ Ntrain } & \multicolumn{2}{|c|}{ NFtrain } & \multicolumn{2}{|c|}{ Ntrain } & \multicolumn{2}{|c|}{ NFtrain } & \multicolumn{2}{|c|}{ Ntrain } & \multicolumn{2}{|c|}{ NFtrain } & \multicolumn{2}{|c|}{ Ntrain } \\
\hline & SFLS & NSFLS & SFLS & NSFLS & SFLS & NSFLS & SFLS & NSFLS & SFLS & NSFLS & SFLS & NSFLS & SFLS & NSFLS & SFLS & NSFLS & SFLS & NSFLS & SFLS & NSFLS \\
\hline \multirow[t]{2}{*}{ NFtest } & 0.0312 & 0.0312 & 0.0312 & 0.0312 & 0.0316 & 0.0316 & 0.0316 & 0.0316 & 0.0370 & 0.0370 & 0.0370 & 0.0370 & 0.0581 & 0.0581 & 0.0581 & 0.0581 & 0.0657 & 0.0657 & 0.0657 & 0.0657 \\
\hline & 0.0312 & 0.0312 & 0.0312 & 0.0312 & 0.0316 & 0.0316 & 0.0316 & 0.0316 & 0.0366 & 0.0366 & 0.0366 & 0.0366 & 0.0710 & 0.0710 & 0.0710 & 0.0710 & 0.0739 & 0.0739 & 0.0739 & 0.0739 \\
\hline \multirow[t]{2}{*}{20} & 0.0424 & 0.0424 & 0.0535 & 0.0534 & 0.0422 & 0.0423 & 0.0531 & 0.0530 & 0.0457 & 0.0457 & 0.0546 & 0.0545 & 0.0600 & 0.0595 & 0.0621 & 0.0623 & 0.0678 & 0.0757 & 0.0701 & 0.075 \\
\hline & 0.0424 & 0.0424 & 0.0535 & 0.0534 & 0.0422 & 0.0423 & 0.0531 & 0.0530 & 0.0449 & 0.0449 & 0.0539 & 0.0538 & 0.0659 & 0.0662 & 0.0679 & 0.0679 & 0.0741 & 0.0771 & 0.0765 & 0.0806 \\
\hline \multirow[t]{2}{*}{16} & 0.0553 & 0.0550 & 0.0715 & 0.0706 & 0.0550 & 0.0548 & 0.0703 & 0.0695 & 0.0572 & 0.0566 & 0.0696 & 0.0691 & 0.0665 & 0.0652 & 0.0762 & 0.0757 & 0.0737 & 0.0792 & 0.0875 & 0.0960 \\
\hline & 0.0553 & 0.0550 & 0.0715 & 0.0706 & 0.0550 & 0.0548 & 0.0705 & 0.0697 & 0.0564 & 0.0559 & 0.0693 & 0.0688 & 0.0698 & 0.0686 & 0.0778 & 0.0771 & 0.0782 & 0.0829 & 0.0942 & 0.094 \\
\hline \multirow[t]{2}{*}{10} & 0.0995 & 0.0914 & 0.1059 & 0.1027 & 0.0929 & 0.0902 & 0.1026 & 0.0996 & 0.0923 & 0.0884 & 0.0966 & 0.0950 & 0.0936 & 0.0848 & 0.1009 & 0.0995 & 0.0990 & 0.0981 & 0.1093 & 0.1167 \\
\hline & 0.0995 & 0.0914 & 0.1059 & 0.1027 & 0.0931 & 0.0905 & 0.1034 & 0.1005 & 0.0921 & 0.0883 & 0.0973 & 0.0957 & 0.0923 & 0.0830 & 0.1004 & 0.0992 & 0.0998 & 0.1045 & 0.1153 & 0.1215 \\
\hline \multirow[t]{2}{*}{4} & 0.3352 & 0.1513 & 0.2030 & 0.1796 & 0.2809 & 0.1481 & 0.1915 & 0.1724 & 0.2231 & 0.1409 & 0.1727 & 0.1615 & 0.1603 & 0.1264 & 0.1593 & 0.1550 & 0.1492 & 0.1431 & 0.1615 & 0.1648 \\
\hline & 0.3352 & 0.1513 & 0.2030 & 0.1796 & 0.2922 & 0.1492 & 0.1948 & 0.1753 & 0.2486 & 0.1425 & 0.1767 & 0.1648 & 0.1541 & 0.1253 & 0.1588 & 0.1548 & 0.1468 & 0.1394 & 0.1626 & 0.1665 \\
\hline \multirow[t]{2}{*}{0} & 0.5149 & 0.2072 & 0.2950 & 0.2373 & 0.4590 & 0.2029 & 0.2738 & 0.2257 & 0.4033 & 0.1934 & 0.2380 & 0.2072 & 0.3105 & 0.1719 & 0.1998 & 0.1814 & 0.2750 & 0.1830 & 0.1987 & 0.1898 \\
\hline & 0.5149 & 0.2072 & 0.2950 & 0.2373 & 0.4803 & 0.2046 & 0.2807 & 0.2305 & 0.4407 & 0.1964 & 0.2492 & 0.2136 & 0.3310 & 0.1728 & 0.2013 & 0.1824 & 0.2720 & 0.1813 & 0.1984 & 0.1910 \\
\hline
\end{tabular}

support of the LMF is constant) and preserves the original shape of the MF (the LMF and UMF keep their original T1 MF shape) for comparison between T1 and IT2 FLSs. The proposed approach (M2) enables both the adaptation of the IT2 FS for known levels of uncertainty (i.e. by increasing FOU size with increasing uncertainty) and the systematic comparison to the original T1 FLS to the resulting new IT2 FLS(s). This method is compared with the IT2 FS creation method M1 which follows a similar creation approach as M2 but does not maintain the MF shape.

In order to assess the viability and explore the behaviour of M2, we conducted detailed performance comparison and evaluation in the context of time series analysis. Both methods were tested under different conditions (Noise free and noisy training data, singleton and non-singleton fuzzification) as well as for triangular and trapezoidal MFs. The results indicate in general FLSs based on M1 outperform those based on M2. However, both methods provide expected performance increases [1] for increasing FOU sizes as uncertainty/noise levels increase. Based on this, it seems that in applications where the systematic transition and comparison from an original T1 FLS is paramount, M2 is preferable, as it maintains the MF shapes. In applications where this level of comparability is not vital however, M1 provides superior levels of performance, while also maintaining a systematic and parametrised increase in FOU size in the face of increasing uncertainty.

As part of future work, we will explore the methodological generation of general type-2 FLSs based on information on levels and distribution of uncertainty in given applications.

\section{REFERENCES}

[1] J. Aladi, C. Wagner, and J. Garibaldi, "Type-1 or interval type-2 fuzzy logic systems -on the relationship of the amount of uncertainty and fou size," in IEEE Int. Conf. on Fuzzy Systems, 2014, pp. 2360-2367.

[2] L. A. Zadeh, "The concept of a linguistic variable and its application to approximate reasoning-I," Information Sciences, vol. 8, no. 3, pp. 199-249, 1975.

[3] J. Mendel, Uncertain rule-based fuzzy logic systems: introduction and new directions. Upper Saddle River, NJ, USA: Prentice-Hall, 2001.

[4] D. Wu and J. M. Mendel, "Uncertainty measures for interval type-2 fuzzy sets," Information Sciences, vol. 177, no. 23, pp. 5378-5393, 2007.

[5] C. Wagner and H. Hagras, "Toward general type-2 fuzzy logic systems based on zSlices," IEEE Trans. Fuzzy Syst., vol. 18, no. 4, pp. 637-660, 2010.

[6] J. M. Mendel and R. B. John, "Type-2 fuzzy sets made simple," IEEE Trans. Fuzzy Syst., vol. 10, no. 2, pp. 117-127, 2002.

[7] S. Coupland and R. John, "Geometric type-1 and type-2 fuzzy logic systems," IEEE Trans. Fuzzy Syst., vol. 15, no. 1, pp. 3-15, 2007.

[8] J. M. Mendel and F. Liu, "On new quasi-type-2 fuzzy logic systems," in IEEE Int. Conf. on Fuzzy Systems, 2008, pp. 354-360.

[9] F. Liu, "An efficient centroid type-reduction strategy for general type-2 fuzzy logic system," Information Sciences, vol. 178, no. 9, pp. 22242236, 2008.

[10] S. Greenfield and R. John, "Optimised generalised type-2 join and meet operations," in IEEE Int. Fuzzy Systems Conf., 2007, pp. 1-6. 


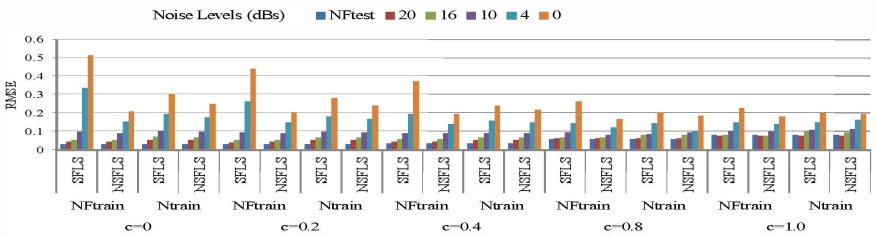

(a)

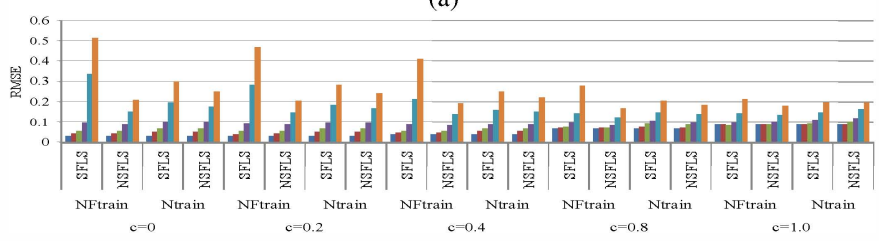

(b)

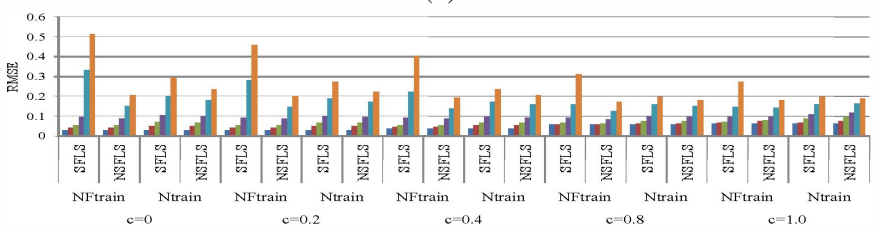

(c)

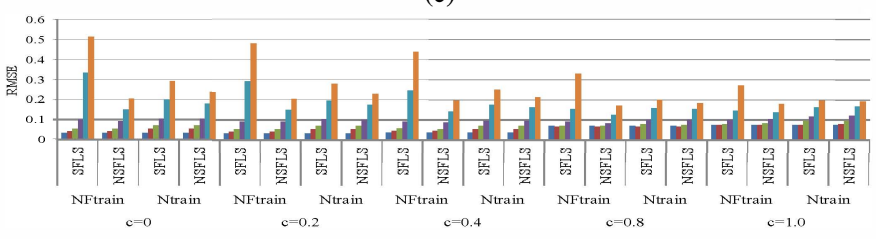

(d)

Fig. 4. (a) The average RMSE results of using triangular MF with M1. (b) The average RMSE results of using triangular MF with M2. (c) The average RMSE results of using trapezoidal MF with M1. (d) The average RMSE results of using trapezoidal MF with M2

[11] C. Wagner and H. Hagras, "zSlices-towards bridging the gap between interval and general type-2 fuzzy logic," in IEEE Int. Conf. on Fuzzy Systems, 2008, pp. 489-497.

[12] J. Mendel, R. John, and F. Liu, "Interval type-2 fuzzy logic systems made simple," IEEE Trans. Fuzzy Syst., vol. 14, no. 6, pp. 808-821, 2006.

[13] N. N. Karnik and J. M. Mendel, "Applications of type-2 fuzzy logic systems to forecasting of time-series," Information Sciences, vol. 120, no. 1, pp. 89-111, 1999.

[14] O. Linda and M. Manic, "Uncertainty-robust design of interval type2 fuzzy logic controller for delta parallel robot," IEEE Trans. Ind. Informat., vol. 7, no. 4, pp. 661-670, 2011.

[15] N. Sahab and H. Hagras, "Adaptive non-singleton type-2 fuzzy logic systems: a way forward for handling numerical uncertainties in real world applications," Int. J. Comput. Commun. Control, vol. 5, no. 3, pp. 503-529, 2011.

[16] N. Benatar, U. Aickelin, and J. M. Garibaldi, "A comparison of nonstationary, type-2 and dual surface fuzzy control," in IEEE Int. Conf. on Fuzzy Systems, 2011, pp. 1193-1200.

[17] J. Mendel and H. Wu, "Type-2 fuzzistics for symmetric interval type-2 fuzzy sets: Part 1, forward problems," IEEE Trans. Fuzzy Syst., vol. 14, no. 6, pp. 781-792, 2006.

[18] J. M. Mendel and H. Wu, "Type-2 fuzzistics for nonsymmetric interval type-2 fuzzy sets: forward problems," IEEE Trans. Fuzzy Syst., vol. 15, no. 5, pp. 916-930, 2007.

[19] C.-H. Lee and H.-Y. Pan, "Performance enhancement for neural fuzzy systems using asymmetric membership functions," Fuzzy Sets and Systems, vol. 160, no. 7, pp. 949-971, 2009.

[20] S. Coupland and R. John, "Geometric interval type-2 fuzzy systems." in EUSFLAT Conf., 2005, pp. 449-454.

[21] M. A. Khanesar, E. Kayacan, M. Teshnehlab, and O. Kaynak, "Analysis of the noise reduction property of type-2 fuzzy logic systems using a novel type-2 membership function," IEEE Trans. Syst., Man, Cybern. B, vol. 41, no. 5, pp. 1395-1406, 2011.

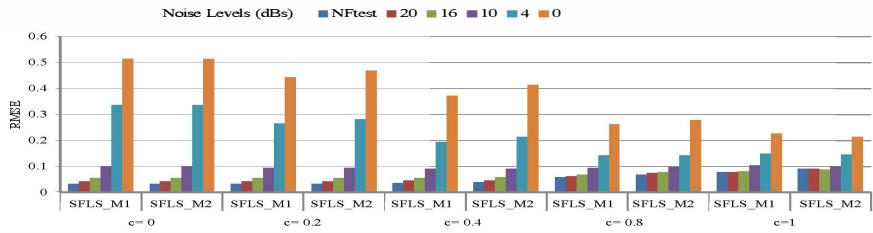

(a)

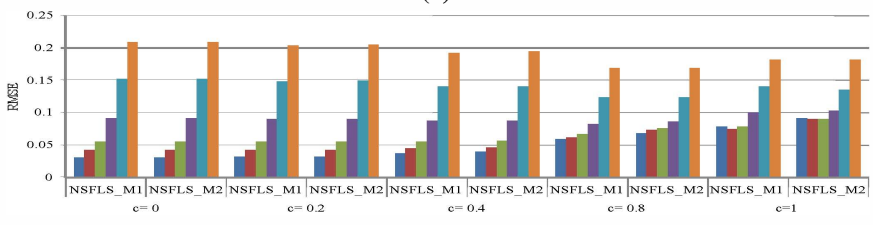

(b)

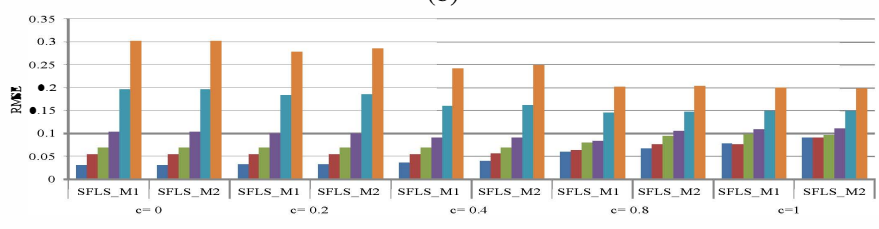

(c)

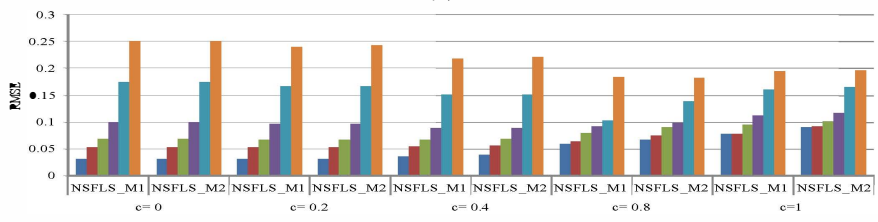

(d)

Fig. 5. Comparison of M1 and M2 performance in case of triangular MF. (a) SFLSs trained on NF data (NFtrain). (b) NSFLSs trained on NF data (NFtrain). (c) SFLSs trained on noisy data (Ntrain). (d) NSFLSs trained on noisy data (Ntrain).

[22] M. Khanesar, M. Teshnehlab, E. Kayacan, and O. Kaynak, "A novel type-2 fuzzy membership function: application to the prediction of noisy data," in IEEE Int. Conf. on Comput. Intell. for Measurement Systems and Applications, 2010, pp. 128-133.

[23] T. Kumbasar and H. Hagras, "A self-tuning zslices based general type-2 fuzzy pi controller," IEEE Trans. Fuzzy Syst., vol. PP, no. 99, p. 1, 2014

[24] N. N. Karnik, J. M. Mendel, and Q. Liang, "Type-2 fuzzy logic systems," IEEE Trans. Fuzzy Syst., vol. 7, no. 6, pp. 643-658, 1999.

[25] N. Sahab and H. Hagras, "Adaptive non-singleton type-2 fuzzy logic systems: a way forward for handling numerical uncertainties in real world applications," Int. J. Comput. Commun. Control, vol. 5, no. 3, pp. 503-529, 2011.

[26] G. C. Mouzouris and J. M. Mendel, "Nonsingleton fuzzy logic systems: theory and application," IEEE Trans. Fuzzy Syst., vol. 5, no. 1, pp. 5671, 1997.

[27] S. Park and H. Lee-Kwang, "A designing method for type-2 fuzzy logic systems using genetic algorithms," in IFSA World Congress and 20th NAFIPS Int. Conf., 2001, pp. 2567-2572.

[28] L. Wang and J. M. Mendel, "Generating fuzzy rules by learning from examples," IEEE Trans. Syst., Man, Cybern., vol. 22, no. 6, pp. 1414 1427, 1992.

[29] M. Versaci and F. C. Morabito, "Fuzzy time series approach for disruption prediction in Tokamak reactors," IEEE Trans. Magn., vol. 39, no. 3, pp. 1503-1506, 2003.

[30] N. N. Karnik and J. M. Mendel, "Applications of type-2 fuzzy logic systems to forecasting of time-series," Information Sciences, vol. 120, no. 1, pp. 89-111, 1999.

[31] J. Jang and C. Sun, "Predicting chaotic time series with fuzzy if-then rules," in Second IEEE Int. Conf. on Fuzzy Systems, 1993, pp. 10791084.

[32] M. C. Mackey and L. Glass, "Oscillation and chaos in physiological control systems," Science, vol. 197, no. 4300, pp. 287-289, 1977.

[33] D. A. Quinney, An Introduction to the Numerical Solution of Differential Equations. New York, NY, USA: John Wiley \& Sons, Inc., 1987. 